\title{
Angular momentum transfer torques in spin valves with perpendicular magnetization
}

\author{
Xingtao Jia, Ying Li, and Ke Xia \\ Department of Physics, Beijing Normal University, Beijing 100875, China \\ Gerrit E. W. Bauer \\ Delft University of Technology, Kavli Institute of NanoScience, 2628 CJ Delft, The Netherlands and \\ Institute for Materials Research, Tohoku University, Sendai 980-8577, Japan
}

(Dated: November 3, 2018)

\begin{abstract}
Spin valves incorporating perpendicularly magnetized materials are promising structures for memory elements and high-frequency generators. We report the angular dependence of the spin-transfer torque in spin valves with perpendicular equilibrium magnetization computed by first-principles circuit theory and compare results with experiments by W.H. Rippard c.s. [Phys. Rev. B 81, 014426 (2010)] on the CoFe $|\mathrm{Cu}| \mathrm{CoNi}$ system. Furthermore, we predict a non-monotonous ("wavy") spin-transfer torque when the $\mathrm{Cu}$ spacer is replaced by a $\mathrm{Ru}$ layer.

PACS numbers: 72.25.Ba, 85.75.-d, 72.10.Bg
\end{abstract}

\section{INTRODUCTION}

A current can be used to read out the information in magnetic memory devices by the giant magnetoresistance. Magnetic random access memory (MRAM) technology has become scalable by writing information using the current-induced spin-transfer torques (STT) $\underline{\underline{1}} \underline{\underline{\underline{4}}}$ The critical electric current density $j_{c}$ necessary to switch a magnetic layer in a spin-valve structure is an allimportant figure of merit in this case. The introduction of materials with perpendicular magnetocrystalline anisotropies that forces the equilibrium magnetization out of the plane,,$\underline{\underline{5}}$ has helped to reduce $j_{c} \underline{\underline{\underline{6}}} \underline{\underline{\underline{8}}}$

$\mathrm{Co} \mid \mathrm{Ni}$ multilayers are an interesting system with per-

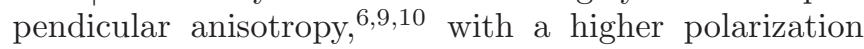
and less spin-flip scattering than, for example, $\mathrm{CoPt}$ alloy $\stackrel{11}{=}$ Rippard et al $\stackrel{12}{n}$ studied current-induced highfrequency generation in structures with a perpendicularly polarized $(\mathrm{Co} \mid \mathrm{Ni})_{n}$ multilayer serving as the switchable magnet and an in-plane magnetized Co layer as polarizer. The output power of such a device depends sensitively on the asymmetry of the angular dependent STT when the magnetization of the free layer is reversed, ${ }^{1,2,13}$ By generating an rf output by a dc current in a spin valve in which the free layer is magnetized normal to the polarizing layer, Rippard c.s. parameterized the skewness of the torque as a function of magnetization angle $\frac{12}{2}$ Koyama et al $\stackrel{14}{n}$ measured high speed current-induced domain wall velocities $(40 \mathrm{~m} / \mathrm{s})$ in magnetic perpendicular $\mathrm{Co} \mid \mathrm{Ni}$ multilayers with current-in-plane configuration. Another interesting materials system is $\mathrm{Co} \mid \mathrm{Ru} \stackrel{15,16}{=}$ which also displays perpendicular magnetic anisotropy 17

Semiclassical theories ${ }^{18,19}$ that combine a quantum treatment of the interface scattering and diffusion treatment of bulk scattering in general explain experiments on magnetic metallic multilayers well ${ }^{2}$ Here we report calculations of the STT of spin valves containing perpendicularly oriented ferromagnetic materials based on magnetoelectronics circuit theory using interface transport parameters computed by first principles. The spin- orbit coupling is the origin of the magnetic crystalline anisotropy and perpendicular magnetization. However, the experimental spin-dependent interface resistances for not too heavy elements can be reproduced by parameterfree calculations without taking into account the spin orbit interaction, 20 which will therefore be disregarded in the following.

Here, we study the angular dependent STT in $\mathrm{Co}_{1} \mathrm{Ni}_{x}$ (the subscripts refer to the number of atomic layers) based spin valves by circuit theory in combination with first-principles calculations. Firstly, we present results for $\mathrm{Co}|\mathrm{Cu}|\left(\mathrm{Co}_{1} \mathrm{Ni}_{x}\right)_{y} \mathrm{Co}_{1} \mid \mathrm{Cu}(111)$ stuctures, where the subscripts 1 and $x$ indicate again the number of atomic layers, while $y$ is the number of stacks and compare them with experiments $\underline{12}$ Next, we report large and 'wavy' angular-dependent STT for $\mathrm{Co}|\mathrm{Ru}|\left(\mathrm{Co}_{1} \mathrm{Ni}_{2}\right)_{x} \mathrm{Co}_{1} \mid \mathrm{Ru}(111)$ spin valves which might therefore be very efficient high-frequency generators.

In Sec. III we introduce our method to calculate the STT in spin valves in terms of the spin mixing conductances of the interfaces computed from first principle, including corrections for the magnetically active bulk material and the diffusive environment. In Sec. [II] we present results for the spin mixing conductances for two the two types of spin vales with perpendicular magnetic anisotropy and compute the angular dependence of STT by magnetoelectronic circuit theory. We summarize our results in Sec. IV]

\section{SPIN MIXING CONDUCTANCE IN A DIFFUSIVE ENVIRONMENT}

The STT due to a current bias $I$ in ferromagnet|normal-metal|ferromagnet $\quad(\mathrm{F}|\mathrm{N}| \mathrm{F})$ spin valves in which the magnetizations are at an angle $\theta$ can be computed analytically by circuit theory ${ }^{2,21}$ and, 
assuming structural symmetry, be parameterized as 13

$$
\tau(\theta)=\frac{\hbar I \tilde{P}}{4 e} \frac{\Lambda \sin \theta}{\Lambda \cos ^{2}(\theta / 2)+\Lambda^{-1} \sin ^{2}(\theta / 2)},
$$

where the asymmetry parameter can be expressed in terms of the the parameters of the $\mathrm{N} \mid \mathrm{F}$ interface as $\Lambda=|\tilde{\eta}| / \sqrt{\left(1-\tilde{P}^{2}\right) \operatorname{Re} \tilde{\eta}}$, where $\tilde{\eta}=2 \tilde{G}_{\uparrow \downarrow} /\left(\tilde{G}_{\uparrow}+\tilde{G}_{\downarrow}\right)$ is the normalized effective spin-mixing conductance and $\tilde{P}=\left(\tilde{G}_{\uparrow}-\tilde{G}_{\downarrow}\right) /\left(\tilde{G}_{\uparrow}+\tilde{G}_{\downarrow}\right)$ is the conductance polarization. Here, $\tilde{G}_{\uparrow}, \tilde{G}_{\downarrow}$ and $\tilde{G}_{\uparrow \downarrow}$ are the spin-dependent and spin-mixing conductances, respectively, where the tilde indicates that they have been "Schep corrected" for a diffusive environment and include the effects of the magnetically active contact regions close to the interface. In deriving Eq. (11) spin flip in the normal layer has been disregarded. When the spin-flip diffusion length in the magnetic layers is much longer than the bulk layer thickness: $\stackrel{2}{2}$

$$
\frac{1}{\tilde{G}_{\sigma}}=\frac{1}{G_{\sigma}}+\frac{1}{2} \frac{e^{2}}{h}\left(\frac{\rho_{F, \sigma} d_{F}}{A_{F}}\right)-\frac{1}{2}\left(\frac{1}{G_{N}^{s h}}+\frac{1}{G_{F, \sigma}^{s h}}\right)
$$

and

$$
\frac{1}{\tilde{G}_{\uparrow \downarrow}}=\frac{1}{G_{\uparrow \downarrow}}-\frac{1}{2 G_{N}^{s h}} .
$$

where $\sigma$ is the spin index, $d_{F(N)}$ the thickness of ferromagnet $F$ or normal metal $N$ layer, $\rho$ the bulk resistivity (for a single spin), and $A_{F}$ the pillar cross section. The $G^{s h}$ 's are Sharvin conductances, $G_{\uparrow}=\left(e^{2} / h\right) \operatorname{tr} \mathbf{t}_{\uparrow}^{\dagger} \mathbf{t}_{\uparrow}$, $G_{\downarrow}=\left(e^{2} / h\right) \operatorname{tr} \mathbf{t}_{\downarrow}^{\dagger} \mathbf{t}_{\downarrow}$ and $G_{\uparrow \downarrow}=\left(e^{2} / h\right) \operatorname{tr}\left(\mathbf{I}-\mathbf{r}_{\uparrow}^{\dagger} \mathbf{r}_{\downarrow}\right)$, where $\mathbf{t}_{\downarrow(\downarrow))}\left(\mathbf{r}_{\uparrow(\downarrow)}\right)$ are the matrices of the transmission (reflection) coefficients of the phase coherent region of the $\mathrm{N} \mid \mathrm{F}$ contact as seen from the normal metal and at the Fermi energy. $\mathbf{I}$ is an $M \times M$ unit matrix, where $M$ is the number of conducting channels in $N$. The third term on the right-hand side of the last two equations are the Schep correction, while the second terms correct for the magnetically active bulk regions. When the ferromagnetic layer is much thicker than the spin-flip diffusion length $l_{s d}^{F}$, the latter should replace $d_{F}$ in Eq. (2). With the spin-orbit interaction we also ignore intrinsic spin-flip scattering at the interfaces. The ferromagnetic layers are assumed sufficiently thick such that mixing transmission contribution may be disregarded. ${ }^{22}$ Note that Eq. (11) only holds for structurally symmetric spin valves. In the following we use the general expression in which the left and right interface parameters differ, as shown in Fig 1 but do not list the expressions explicitly here (see Refs. 21, 23 25).

In our calculations the atomic potentials were determined in the framework of the tight-binding (TB) linear muffin-tin- orbital (MTO) method 26 based on density functional theory in the local density approximation

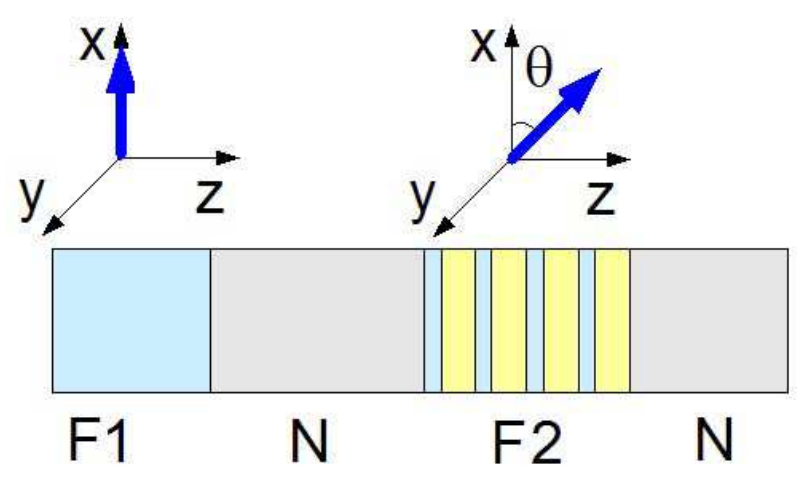

FIG. 1: Scheme of asymmetric $\mathrm{F} 1|\mathrm{~N}| \mathrm{F} 2 \mid \mathrm{N}$ spin valves with perpendicular magnetization F2 used in the calculations

and an exchange-correlation potential parameterized by von Barth and Hedin. 27 The self-consistent crystal potentials were used as input to a TB-MTO wave-functionmatching calculation, from which we obtained the transmission and reflection at the interfaces. The calculations are carried out with a $\mathbf{k}_{\| \mid}$mesh density equivalent to more than $3600 \mathbf{k}_{\|}$mesh points in the two dimensional Brillouin zone (BZ) corresponding to the interface unit cell. The technical detail can be found in Ref. 28. Table \ compiles our results for various interface conductances including the bulk corrections due to magnetically active regions.

\section{III. $\mathrm{CO}_{1} \mathrm{NI}_{2} \mid \mathrm{CU}$ AND $\mathrm{CO}_{1} \mathrm{NI}_{2} \mid \mathrm{RU}$ MULTILAYERS}

We first focus on the Co|Ni multilayers, which we treat as phase coherent regions, i.e. we compute the scattering matrix of the entire multilayers, which is then treated in the circuit theory of conventional spin valves just like a single interface. We present the spindependent and mixing conductances of $\mathrm{Cu}\left|\mathrm{X}_{n}\right| \mathrm{Cu}$ with $\left[\mathrm{X}_{n}=\left(\mathrm{Co}_{1} \mathrm{Ni}_{2}\right)_{n} \mathrm{Co}_{1}\right]$. Here the $\mathrm{Cu}$ leads on both sides are semi-infinite. $\mathrm{X}_{n}$ denotes $n$ repetitions of the $\mathrm{Co}_{1} \mathrm{Ni}_{2}$ multilayer unit. As in the experiments $\stackrel{12}{=}$ a Co atomic layer is added for better contact with the $\mathrm{Cu}$ reservoirs. Since samples have been grown by sputtering, we take interface disorder into account, which is in general well modeled by a two monolayer $50 \%-50 \%$ interfacial alloy $\left(\mathrm{Co}_{1} \mathrm{Ni}_{2}\right)_{n} \rightarrow\left(\left[\mathrm{Co}_{0.5} \mathrm{Ni}_{0.5}\right] \mathrm{Ni}\left[\mathrm{Co}_{0.5} \mathrm{Ni}_{0.5}\right]\right)_{n} .2$ Spin-flip scattering at the $\mathrm{Co} \mid \mathrm{Ni}$ interface will suppress any benefits of an even larger number of $\mathrm{Co} \mid \mathrm{Ni}$ interfaces $\stackrel{20}{=} \mathrm{We}$ therefore present here only calculations with $n \leq 5$. The computed dimensionless mixing conductance $\tilde{\eta}$ is also listed in the table.

In the fcc crystal structure Co and Ni have nearly identical band structures for the majority spin, which results in very transparent $\mathrm{Co} \mid \mathrm{Ni}$ interfaces. The majority spin conductance therefore stays nearly constant with increasing $n$. For minority spin electrons the scattering at the 
FIG. 2: Comparison of computed and experimental (Ref. 12) angular dependent STT in $\mathrm{F} 1|\mathrm{Cu}|\left(\mathrm{Co}_{1} \mathrm{Ni}_{2}\right)_{5} \mathrm{Co}_{1} \mid \mathrm{Cu}(\mathrm{F} 1=\mathrm{Co}$, $\left.\mathrm{Co}_{90} \mathrm{Fe}_{10}\right)$ spin valves with two monolayer $50 \%-50 \%$ intermixed interfaces. For Co as fixed lead, we vary the thickness $d_{\text {Co }}$ from $5 \mathrm{~nm}$ to $20 \mathrm{~nm}$. When using $\mathrm{Cog}_{90} \mathrm{Fe}_{10}$ as fixed layer, we use $d_{\mathrm{Cog0}_{0} \mathrm{Fe}_{10}}=2.5 \mathrm{~nm}$, and resistivity $\rho_{\mathrm{Cog}_{90} \mathrm{Fe}_{10}}=$ $154 \Omega \mathrm{nm}$ (Ref. 29) and $\mathrm{Co} \mid \mathrm{Cu}$ interface parameters. The dark area indicated the experiment results parameterized by Slonczewski's formula with $\Lambda=1.3$ and $\Lambda=1.7$. Calculations are carried out by circuit theory for an asymmetric spin valve with first-principles interface parameters using the Schep correction including the contribution form the magnetically active region of the bulk ferromagnet as described in the text.

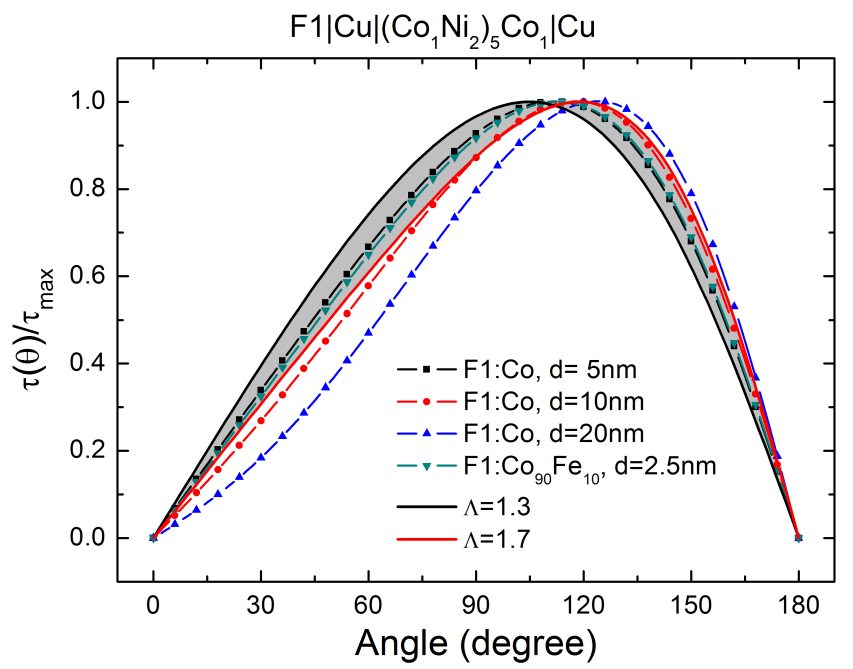

$\mathrm{Co} \mid \mathrm{Ni}$ interface is much stronger. Consequently the minority spin conductance decreases rapidly with increasing number of $\mathrm{Co} \mid \mathrm{Ni}$ interfaces.

Fig. 2 shows the angular dependent STT exerted on the right-hand side of $\mathrm{F} 1|\mathrm{Cu}|\left(\mathrm{Co}_{1} \mathrm{Ni}_{2}\right)_{5} \mathrm{Co}_{1} \mid \mathrm{Cu}(\mathrm{F} 1=\mathrm{Co}$, $\left.\mathrm{Co}_{90} \mathrm{Fe}_{10}\right)$ spin valves with intermixed interfaces calculated by our first-principles circuit theory and compared with the experimental result $\underline{\underline{12}}$ For pure Co as fixed lead, we vary $d_{\text {Co }}$ from $5-20 \mathrm{~nm}$ and find that the angular dependent STT falls into the experimental range 12 estimated by Slonczewski's formula for symmetric spin valves with $\Lambda=1.3$ and 1.7. Experimentally, $\mathrm{Co}_{90} \mathrm{Fe}_{10}$ is used as fixed layer. Its spin-flip diffusion length is shorter than that of Co, but its resistivity is also higher, so there is not much difference when compared with a Co polarizer. We assume that the interface is not affected. We plot the results of $\mathrm{CoFe}$ in Fig. 2 with $d_{\mathrm{Co}_{90} \mathrm{Fe}_{10}}=2.5 \mathrm{~nm}$ (Ref. 31), and $\rho_{\mathrm{Cog}_{90} \mathrm{Fe}_{10}}=154 \Omega \mathrm{nm}$ and is very similar to pure Co with $d_{\mathrm{Co}}=5 \mathrm{~nm}$. The results for $\mathrm{Co}|\mathrm{Cu}|\left(\mathrm{Co}_{1} \mathrm{Ni}_{x}\right)_{y} \mathrm{Co}_{1} \mid \mathrm{Cu}$ from $x=2-3$ and $y=2-5$ are shown in Fig. 3. We observe large difference between epitaxial and disordered samples, but only weak dependences on $x$ and $y$. The results for epitaxial (disordered) samples fall into the range of Slonczewski's $\Lambda=1.05-1.15(1.4-1.5)$.

The experimental results were parameterized by Eq.

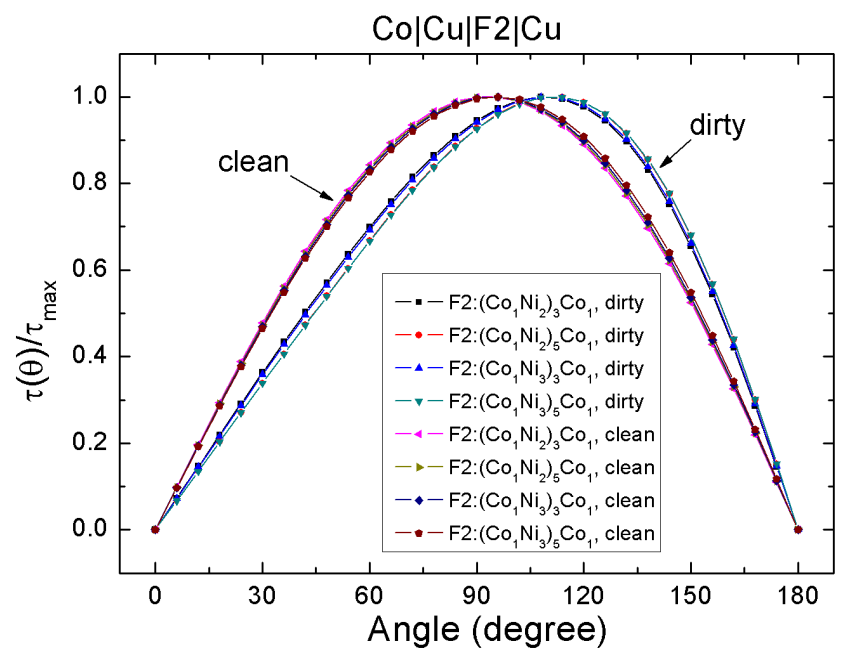

FIG. 3: Angular dependent STT in $\mathrm{Co}|\mathrm{Cu}|\left(\mathrm{Co}_{1} \mathrm{Ni}_{2}\right)_{x} \mathrm{Co}_{1} \mid \mathrm{Cu}$ and $\mathrm{Co}|\mathrm{Cu}|\left(\mathrm{Co}_{1} \mathrm{Ni}_{3}\right)_{x} \mathrm{Co}_{1} \mid \mathrm{Cu}$ spin valves with $d_{\mathrm{Co}}=5 \mathrm{~nm}$.

(11) for a structurally symmetric spin valve, whereas our results are based on the theory for asymmetric structures $\stackrel{21}{\underline{21}}$ We suggest that in future experiments Slonczewski's formula should be replaced by a more accurate parameterization.

Another interesting material with perpendicular magnetic anisotropy is $\mathrm{Co} / \mathrm{Ru}$. Experimentally, both $\mathrm{hcp}(0001)^{33}$ and $\mathrm{fcc}(111)^{\frac{34}{4}}$ structures have been reported. Despite the large lattice mismatch between Co and $\mathrm{Ru}$, hcp $\mathrm{Co} \mid \mathrm{Ru}$ could be grown epitaxially and the magnetic anisotropy depends on the thickness of the Co layer $\stackrel{35}{ }$ However, the metastable structure relaxes to a more stable one after annealing $\underline{35} \mathrm{Co}|\mathrm{Ru}| \mathrm{Co}$ with a metastable fcc(111) structure has also been reported. $\underline{36}$ Here we present systematic calculations of the transport properties of $\mathrm{Co} \mid \mathrm{Ru}$ pillars with different structure and lattice constants as listed in Table II.

For epitaxial samples, we show results for an fcc(111) texture with lattice parameters for $\mathrm{Ru}, \mathrm{Co}$, and its average. The lattice parameter along the growth direction is varied to keep the atomic volume constant. Both spin polarization and specific resistance are close to the experimental values $\stackrel{34}{=}$ but considering the large lattice distortion $(7.3 \sim 14 \%)$ this may be accidental.

For the epitaxial hcp(0001) texture our calculations yield very high spin polarizations $\tilde{P}=-39 \sim-55 \%$ for both clean and dirty interfaces when Co adopts the $\mathrm{Ru}$ structure and lattice constants as reported $\frac{38}{\underline{G}}$ and small specific resistances $A \tilde{R}=A / \tilde{G}=0.69-0.78 \times$ $10^{-15} \Omega \mathrm{m}^{2}$. Here and below $\tilde{P}$ and $\tilde{G}$ have been Schep corrected with magnetically active layer thickness $d_{\text {Co }}=$ $5 \mathrm{~nm}$. Note that the structure is metastable and under annealing Co is expected to return to its normal lattice parameter.

To simulate sputtering conditions, a $14 \times 14$ Co is matched to a $13 \times 13 \mathrm{Ru}$ lateral super-cell for both fcc(111) and hcp(0001), leading to a spin polarization 

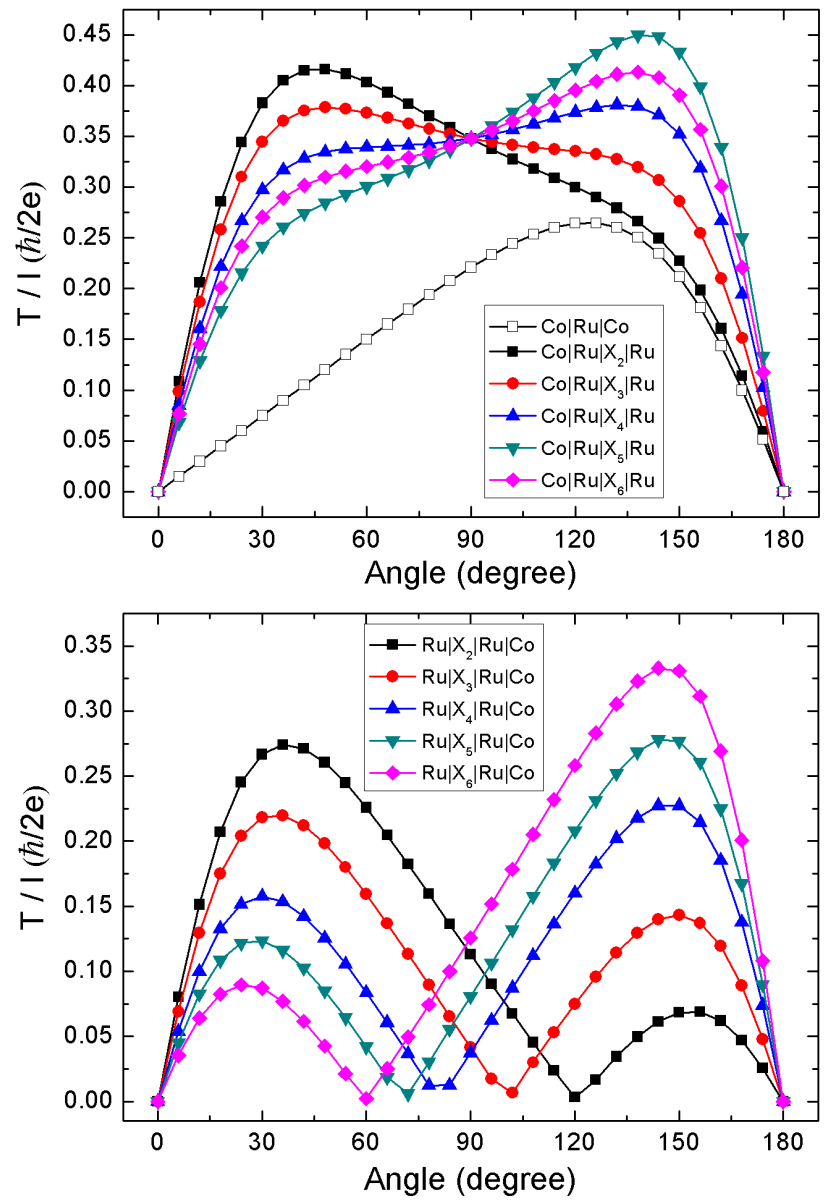

FIG. 4: Angular-dependent torkance $T / I$ on the rightside ferromagnet in disordered $\mathrm{Ru}$ based spin valves with $\mathrm{X}_{n}=\left(\mathrm{Co}_{1} \mathrm{Ni}_{2}\right)_{n} \mathrm{Co}_{1}$. The Co and $\mathrm{Ru}$ buffer layers are assumed much thicker than the spin diffusion lengths $l_{s d}^{\mathrm{Co}}$ and $l_{s d}^{\mathrm{Ru}}$, so that the Schep correction includes the bulk scattering of the latter length scales. We disregard the bulk scattering in the $\mathrm{Ru}$ spacer, which should be allowed for the small thickness of 8 monolayer $(2.21 \mathrm{~nm})$ considered.

of $\tilde{P}=-15 \%$ and specific resistance of $A R=0.75 \times$ $10^{-15} \Omega \mathrm{m}^{2}$ for a clean fcc(111) texture, $\tilde{P}=-28 \%$ and $A \tilde{R}=0.93 \times 10^{-15} \Omega \mathrm{m}^{2}$ for a clean hcp $(0001)$ texture. A $50 \%-50 \%$ interface alloy has little effect on fcc(111) texture, but leads to a reduced $\tilde{P}=-19 \%$ for the hcp (0001) texture. The measured spin polarization for the Co $\mid \mathrm{Ru}$ interface is $\tilde{P}=-20 \%$ with specific resistance $A \tilde{R}=0.5 \times 10^{-15} \Omega \mathrm{m}^{2}$ (Ref. ? ).

In Table I we observe that in contrast to the $\mathrm{Co} / \mathrm{Cu}$ interface, $\mathrm{Co} \mid \mathrm{Ru}$ has a negative spin polarization for both fcc(111) and hcp(0001) orientations. Interesting is the relatively large dimensionless mixing conductance $\tilde{\eta}$. The predicted very large mixing conductance implies a large skewness of the angular dependent STT, which makes this material promising for applications in high-frequency generators.

Fig. 4 gives the angular dependent $T / I$ in $\mathrm{Co}|\mathrm{Ru}| \mathrm{FM} \mid \mathrm{Ru}(111)$ spin valves. Here, disorder is modeled again by two monolayers of a $50 \%-50 \%$ interface alloy (to rather small effect) and Schep and magnetic bulk corrections have been implemented. When fitted by Slonczewski's formula, the STT on the soft $\mathrm{Co}_{1} \mathrm{Ni}_{2}$ multilayer in the strongly asymmetric spin valve $\mathrm{Co}|\mathrm{Ru}|\left(\mathrm{Co}_{1} \mathrm{Ni}_{2}\right)_{y} \mathrm{Co}_{1} \mid \mathrm{Ru}(111)$ shows a large variation in skewness in terms of the parameter $\Lambda=0.5-2.0$. The maximum of the angular dependent spin torque is shifted gradually from low angle to high angle when the thickness of $\mathrm{Co}_{1} \mathrm{Ni}_{2}$ increases from 2 to 6 periods. When $\mathrm{Co}$ serves as the free layer, the (modulus of the) angular dependent torkance shows two peaks and a compensation point when the thickness of $\mathrm{Co}_{1} \mathrm{Ni}_{2}$ increases from 3 to 5 periods. This shape can be understood in terms of the spin accumulation in the normal metal spacer in the parallel configuration, $\stackrel{40}{=}$ which is accompanied by a non-monotonic angular magnetoresistance. This behavior has been observed in $\mathrm{Py}|\mathrm{Cu}| \mathrm{Co}$ and dubbed 'wavy torques' ${ }^{\underline{41}, 42}$

\section{SUMMARY}

We studied the angular dependent STT for materials with magnetization normal to the interfaces by circuit theory in combination with first-principles calculations. An interesting angular dependent STT is found in $\mathrm{Co}|\mathrm{Ru}|\left(\mathrm{Co}_{1} \mathrm{Ni}_{2}\right)_{x} \mathrm{Co}_{1} \mid \mathrm{Ru}(111)$ spin valve. Moreover, a 'wavy' angular-dependent STT acts on the Co layer in $\mathrm{Co}|\mathrm{Ru}|\left(\mathrm{Co}_{1} \mathrm{Ni}_{2}\right)_{x} \mathrm{Co}_{1} \mid \mathrm{Ru}(111)$ structures. When the $\mathrm{CoNi}$ is the free layer, we expect very efficient highfrequency generation.

\section{ACKNOWLEDGEMENTS}

The authors acknowledge $\mathrm{Y}$. $\mathrm{Xu}$ and $\mathrm{S}$. Wang for their preliminary work. The authors gratefully acknowledge financial support from National Basic Research Program of China (973 Program) under the grant No. 2011CB921803, and NSF-China grant No. 60825404, the Dutch FOM Foundation, and EU-ICT-7 contract no. 257159 MACALO.
1 J. Slonczewski, J. Magn. Magn. Mater. 159, L1 (1996).

2 A. Brataas, G. E. W. Bauer, and P. J. Kelly, Phys. Rep.
427, 157 (2006)

3 L. Berger, Phys. Rev. B 54, 9353 (1996). 


\begin{tabular}{|c|c|c|c|c|c|c|}
\hline system & $G_{\uparrow}$ & $G_{\downarrow}$ & $\operatorname{Re} G_{\uparrow \downarrow}$ & $\operatorname{Im} G_{\uparrow \downarrow}$ & $\tilde{P}$ & $\tilde{\eta}$ \\
\hline $\mathrm{Cu}\left|\mathrm{X}_{2}\right| \mathrm{Cu}$ & $0.41(0.41)$ & $0.35(0.19)$ & $0.55(0.54)$ & $-0.02(-0.03)$ & $0.25(0.69)$ & $0.85(1.1)$ \\
\hline $\mathrm{Cu}\left|\mathrm{X}_{3}\right| \mathrm{Cu}$ & $0.41(0.41)$ & $0.32(0.18)$ & $0.56(0.54)$ & $-0.03(-0.03)$ & $0.36(0.72)$ & $0.96(1.1)$ \\
\hline $\mathrm{Cu}\left|\mathrm{X}_{4}\right| \mathrm{Cu}$ & $0.41(0.41)$ & $0.31(0.16)$ & $0.56(0.54)$ & $-0.03(-0.03)$ & $0.39(0.75)$ & $0.98(1.2)$ \\
\hline $\mathrm{Cu}\left|\mathrm{X}_{5}\right| \mathrm{Cu}$ & $0.41(0.41)$ & $0.30(0.15)$ & $0.55(0.54)$ & $-0.03(-0.03)$ & $0.42(0.77)$ & $0.97(1.2)$ \\
\hline $\mathrm{Cu}\left|\mathrm{Y}_{2}\right| \mathrm{Cu}$ & $0.39(0.40)$ & $0.30(0.21)$ & $0.40(0.54)$ & $-0.02(-0.03)$ & $0.34(0.62)$ & $0.62(1.2)$ \\
\hline $\mathrm{Cu}\left|\mathrm{Y}_{3}\right| \mathrm{Cu}$ & $0.39(0.39)$ & $0.26(0.19)$ & $0.39(0.54)$ & $-0.02(-0.03)$ & $0.46(0.64)$ & $0.66(1.3)$ \\
\hline $\mathrm{Cu}\left|\mathrm{Y}_{4}\right| \mathrm{Cu}$ & $0.39(0.39)$ & $0.24(0.17)$ & $0.40(0.54)$ & $-0.01(-0.03)$ & $0.52(0.69)$ & $0.71(1.3)$ \\
\hline $\mathrm{Ru} \mid \mathrm{Co}$ & $0.32(0.29)$ & $0.58(0.53)$ & $0.92(0.88)$ & $0.001(0.02)$ & $-0.15(-0.17)$ & $8.9(8.7)$ \\
\hline $\mathrm{Ru}\left|\mathrm{X}_{2}\right| \mathrm{Ru}$ & $0.25(0.25)$ & $0.36(0.31)$ & $1.03(0.94)$ & $-0.02(0.02)$ & $-0.26(-0.15)$ & $4.8(4.6)$ \\
\hline $\mathrm{Ru}\left|\mathrm{X}_{3}\right| \mathrm{Ru}$ & $0.25(0.25)$ & $0.35(0.27)$ & $1.03(0.94)$ & $-0.02(0.02)$ & $-0.24(-0.05)$ & $4.9(5.1)$ \\
\hline $\mathrm{Ru}\left|\mathrm{X}_{4}\right| \mathrm{Ru}$ & $0.25(0.25)$ & $0.33(0.23)$ & $1.03(0.94)$ & $-0.02(0.02)$ & $-0.19(0.06)$ & $5.2(5.7)$ \\
\hline $\mathrm{Ru}\left|\mathrm{X}_{5}\right| \mathrm{Ru}$ & $0.25(0.25)$ & $0.31(0.22)$ & $1.03(0.94)$ & $-0.02(0.02)$ & $-0.15(0.08)$ & $5.5(5.8)$ \\
\hline $\mathrm{Ru}\left|\mathrm{X}_{6}\right| \mathrm{Ru}$ & $0.25(0.25)$ & $0.32(0.20)$ & $1.03(0.94)$ & $-0.02(0.02)$ & $-0.19(0.14)$ & $5.2(6.2)$ \\
\hline hex-Ru|Co & $0.20(0.23)$ & $0.53(0.32)$ & $0.83(0.71)$ & $-0.01(0.01)$ & $-0.28(-0.19)$ & $10(7.9)$ \\
\hline $\mathrm{Cu} \mid \mathrm{Co}^{*}$ & $0.42(0.42)$ & $0.36(0.33)$ & $0.41(0.55)$ & $0.01(0.03)$ & $0.51(0.54)$ & $1.2(2.0)$ \\
\hline
\end{tabular}

${ }^{*}$ Ref 30

TABLE I: Parameters for clean (disordered) interfaces (in units of $\left.10^{15} \Omega^{-1} \mathrm{~m}^{-2}\right) . \mathrm{X}_{n}=\left(\mathrm{Co}_{1} \mathrm{Ni}_{2}\right)_{n} \mathrm{Co}_{1} ; \mathrm{Y}_{n}=\left(\mathrm{Co}_{1} \mathrm{Ni}_{3}\right)_{n} ;$ fcc $\mathrm{Cu}$ and $\mathrm{Ru}$ have Sharvin conductances of $G_{\mathrm{Cu}}^{s h}=0.55 \times 10^{15} \Omega^{-1} \mathrm{~m}^{-2}$ and $G_{\mathrm{Ru}(f c c)}^{s h}=0.98 \times 10^{15} \Omega^{-1} \mathrm{~m}^{-2}$, respectively. $G_{\mathrm{Co}(f c c), \uparrow}^{s h}=0.47$ and $G_{\mathrm{Co}(f c c), \downarrow}^{s h}=1.09 \times 10^{15} \Omega^{-1} \mathrm{~m}^{-2}$ for majority and minority spin in fcc Co, respectively. For hex Ru's $G_{\mathrm{Ru}(h e x)}^{s h}=0.80 \times 10^{15} \Omega^{-1} \mathrm{~m}^{-2}$ and Co with hex Ru structure $G_{\mathrm{Co}(\text { hex }) \uparrow}^{s h}=0.40$ and $G_{\mathrm{Co}(\text { hex }), \downarrow}^{s h}=0.80 \times 10^{15} \Omega^{-1} \mathrm{~m}^{-2}$ for majority and minority spins, respectively. A magnetically active bulk region correction is implemented for the normalized spin polarization $\tilde{P}$ and relative mixing conductance $\tilde{\eta}$. For $\mathrm{Cu} \mid$ Co interface, we use $d_{\mathrm{Co}}=5 \mathrm{~nm}$ (Ref. 12), and bulk resistivity $\rho_{\text {Co }}=60 \Omega \mathrm{nm}$ with spin asymmetry $\beta=0.46$, which results in $\rho_{\text {Co }}^{\uparrow}=81 \Omega \mathrm{nm}$ and $\rho_{\text {Co }}^{\downarrow}=219 \Omega \mathrm{nm}$ (Ref 31 ). We use a spin diffusion length $l_{s d}^{\mathrm{Co}}=60 \mathrm{~nm}$.

\begin{tabular}{|c|c|c|c|}
\hline system & lattice & $\tilde{P}$ & $A R\left(10^{-15} \Omega \mathrm{m}^{2}\right)$ \\
\hline fcc(111) & $\mathrm{Ru}$ & $-0.12(-0.05)$ & $0.56(0.83)$ \\
\hline $\operatorname{fcc}(111)$ & Co & $-0.14(-0.20)$ & $0.67(0.93)$ \\
\hline $\operatorname{fcc}(111)$ & $(\mathrm{Ru}+\mathrm{Co}) / 2$ & $-0.09(-0.02)$ & $0.60(0.87)$ \\
\hline $\operatorname{fcc}(111)$ & Matching* & $-0.15(-0.17)$ & $0.75(0.86)$ \\
\hline hcp(0001) & $\mathrm{Ru}^{\dagger}$ & $-0.55(-0.39)$ & $0.78(0.69)$ \\
\hline hcp $(0001)$ & Matching* & $-0.28(-0.19)$ & $0.93(0.98)$ \\
\hline exper ${ }^{34}$ & & -0.2 & 0.5 \\
\hline
\end{tabular}

${ }^{*} 14 \times 14$ Co matched to $13 \times 13 \mathrm{Ru}^{33,37}$

${ }^{\dagger}$ Cobalt's atomic volume expanded to that of $\mathrm{Ru}$.

TABLE II: Comparison of the calculated spin polarization $\tilde{P}$ (we use $d_{C o}=5 \mathrm{~nm}$ ) and specific interface resistances $A \tilde{R}=A / \tilde{G}$ of clean (disordered) $\mathrm{Co} \mid \mathrm{Ru}$ for different lattice parameters with experiment.

${ }^{4}$ E. B. Myers, D. C. Ralph, J. A. Katine, R. N. Louie, R. A. Buhrman, Science 285, 867 (1999).

5 A. D. Kent, B. Ozyilmaz, and E. del Barco, Appl. Phys. Lett. 84, 3897 (2004).

${ }^{6}$ S. Mangin, D. Ravelosona, J. A. Katine, M.J.Carey, B. D. Terris, and E. E. Fullerton, Nat. Mater., 5, 210 (2006).

7 S. Mangin, Y. Henry, D. Ravelosona, J. A. Katine, and E. E. Fullerton, Appl. Phys. Lett. 94, 012502 (2009).

8 H. Yoda et al., Current Applied Physics 10, e87 (2010).

9 G. H. O. Daalderop, P. J. Kelly and F. J. A. den Broeder, Phys. Rev. Lett. 68, 682 (1992).

10 W. Chen, J. M. L. Beaujour, G. de Loubens, A. D. Kent and J. Z. Sun, Appl. Phys. Lett. 92, 012507 (2008).

11 K. Inomata and Y. Saito, Appl. Phys. Lett. 73, 1143

(1998).

12 W. H. Rippard, A. M. Deac, M. R. Pufall, J. M. Shaw, M. W. Keller, S. E. Russek, G. E. W. Bauer and C. Serpico, Phys. Rev. B 81, 014426 (2010).

13 J. Slonczewski, J. Magn. Magn. Mater. 247324 (2002).

14 T. Koyama, G. Yamada, H. Tanigawa, S. Kasai, N. Ohshima, S. Fukami, N. Ishiwata, Y. Nakatani, T. Ono, Appl. Phys. Express, 1, 101303 (2008).

15 S. S. P. Parkin, N. More, and K. P. Roche, Phys. Rev. Lett. 64, 2304 (1990).

16 C. Song, X. X. Wei, K. W. Geng, F. Zeng, and F. Pan, Phys. Rev. B 72, 184412 (2005).

17 J. Miyawaki, D. Matsumura, H. Abe, T. Ohtsuki, E. Sakai, K. Amemiya, and T. Ohta, Phys. Rev. B 80, 020408(R)(2009).

18 A. Brataas, Yu. V. Nazarov, and G. E. W. Bauer, Phys. Rev. Lett. 84, 2481 (2000); Eur. Phys. J. B 22, 99 (2001).

19 M. D. Stiles and A. Zangwill, Phys. Rev. B 66, 014407 (2002).

20 H. Y. T. Nguyen, R. Acharyya, E. Huey, B. Richard, R. Loloee, W. P. Pratt, Jr., J. Bass, S. Wang and K. Xia, Phys. Rev. B 82, 220401(R) (2010).

21 A. A. Kovalev, A. Brataas, and G. E. W. Bauer, Phys. Rev. B 66, 224424 (2002).

22 A. A. Kovalev, A. Brataas, and G. E. W. Bauer, Phys. Rev. B 73, 054407 (2006).

23 J. Xiao, A. Zangwill, and M. D. Stiles, Phys. Rev. B 70, 172405 (2004).

24 J. Barnas, A. Fert, M. Gmitra, I. Weymann, and V. K. Dugaev, Phys. Rev. B 72, 024426 (2005).

25 V. S. Rychkov, S. Borlenghi, H. Jaffres, A. Fert, and X. Waintal, Phys. Rev. Lett. 103, 066602 (2009).

${ }^{26}$ I. Turek, V. Drchal, J. Kudrnovsky, M. Sob, and P. Wein- 
berger, Electronic structure of Disordered Alloys, Surfaces and Interfaces (Kluwer, Boston-London-Dordrecht, 1997).

27 U. von Barth and L. Hedin, J. Phys. C 5, 1629 (1972).

${ }^{28}$ K. Xia, M. Zwierzycki, M. Talanana, P. J. Kelly, and G. E. W. Bauer, Phys. Rev. B 73, 064420 (2006).

29 Y. Jiang, S. Abe, T. Nozaki, N. Tezuka, and K. Inomata, Phys. Rev. B 68, 224426 (2003).

${ }^{30}$ K. Xia, P. J. Kelly, G. E. W. Bauer, A. Brataas, and I. Turek, Phys. Rev. B, 65, 220401(R) (2002).

31 J. Bass, and W. P. Pratt, Jr., J. Magn. Magn. Mater. 200, 274 (1999).

${ }^{32}$ K. Satoshi, A.Yasuo, M. Terunobu, and M. Shigemi, Jpn. J. Appl. Phys. 45, 3892 (2006).

33 F. E. Gabaly, J. M. Puerta, C. Klein, A. Saa, A. K. Schmid, K. F. McCarty, J. I. Cerda, and J. de la Figuera, New J. Phys. 9, 80 (2007).

${ }^{34}$ K. Eid, R. Fonck, M. A. Darwish, W. P. Pratt, Jr., and J. Bass, J. Appl. Phys. 91, 8102 (2002), C. Ahn, K.-H. Shin, and W. P. Pratt, Jr., Appl. Phys. Lett. 92, 102509 (2008).

35 C. Liu and S.D. Bader, J. Magn. Magn. Mater. 119, 81
(1993).

36 P. J. H. Bloemen, H. W. van Kesteren, H. J. M. Swagten, and W. J. M. de Jonge, Phys. Rev. B. 50, 13505 (1994).

37 M. Grimsditch, J. E. Mattson, C. H. Sowers, S. D. Bader, and M. J. Peters, Phys. Rev. Lett. 77, 2025 (1996).

38 K. Rahmouni, A. Dinia, D. Stoeffler, K. Ounadjela, H. A. M. van den Berg, and H. Rakoto, Phys. Rev. B. 59, 9475 (1999).

39 Y. Tserkovnyak, A. Brataas, and G. E. W. Bauer, Phys. Rev. B 66, 224403 (2002).

40 J. Manschot, A. Brataas, and G. E. W. Bauer, Phys. Rev. B 69, 092407 (2004).

41 O. Boulle, V. Cros, J. Grollier, L. G. Pereira, C. Deranlot, F. Petroff, G. Faini, J. Barnas, and A. Fert, Nat. Phys. 3, 492 (2007).

42 O. Boulle, V. Cros, J. Grollier, L. G. Pereira, C. Deranlot, F. Petroff, G. Faini, J. Barnas, and A. Fert, Phys. Rev. B, 77, 174403 (2008). 